SLAC-PUB-10334

February 2004

\title{
Suppression of Microbunching Instability in the Linac Coherent Light Source*
}

\author{
Z. Huang,,${ }^{1}{ }^{\dagger}$ M. Borland,${ }^{2}$ P. Emma,${ }^{1}$ J. Wu,${ }^{1}$ C. Limborg, ${ }^{1}$ G. Stupakov, ${ }^{1}$ J. Welch ${ }^{1}$ \\ ${ }^{1}$ Stanford Linear Accelerator Center, Menlo Park, CA 94025 \\ 2 Argonne National Laboratory, Argonne, IL 60439
}

\begin{abstract}
A microbunching instability driven by longitudinal space charge, coherent synchrotron radiation, and linac wakefields is studied for the linac coherent light source (LCLS) accelerator system. Since the uncorrelated (local) energy spread of electron beams generated from a photocathode rf gun is very small, the microbunching gain may be large enough to significantly amplify rf-gun generated modulations or even shot-noise fluctuations of the electron beam. The uncorrelated energy spread can be increased by an order of magnitude to provide strong Landau damping against the instability without degrading the free-electron laser performance. We study different damping options in the LCLS and discuss an effective laser heater to minimize the impact of the instability on the quality of the electron beam.
\end{abstract}

Submitted to Phys. Rev. ST Accel. Beams

*Work supported by Department of Energy contract DE-AC03-76SF00515 and W-31-109-ENG-38.

$\dagger^{\dagger}$ Email: zrh@slac.stanford.edu 


\section{INTRODUCTION}

An x-ray free electron laser (FEL) is the primary candidate for a fourth-generation light source that provides extremely bright x-ray photons with femtosecond time resolution $[1,2]$. In order to reach the desired electron peak current capable of inducing the collective FEL instability, the pulse length of a low-emittance electron bunch generated from the photocathode rf gun is magnetically compressed in the linear accelerator by more than one order of magnitude. Numerical and theoretical investigations of high-brightness electron bunch compression lead to a microbunching instability driven by coherent synchrotron radiation (CSR) that can significantly degrade the beam quality [3-6]. Recently, Saldin et al. point out that the longitudinal space charge (LSC) field can be the main effect driving the microbunching instability in the TESLA test facility (TTF) (phase 2) linac [7]. In addition, significant LSC-induced energy modulation in the DUV-FEL linac has been experimentally characterized using an rf zero-phasing method [8]. Because the microbunching instability is very sensitive to the uncorrelated (local) energy spread of the electron beam, increasing it within the FEL tolerance can provide strong Landau damping against the instability. Possible solutions include the use of a superconducting (SC) wiggler [1] or the resonant laser-electron interaction in an undulator (a laser heater) [7, 9].

In this paper, we study the suppression of the microbunching instability including LSC, CSR, and linac wakefields in the linac coherent light source (LCLS). In Sec. II, we discuss the microbunching gain with and without the SC wiggler. We find that the wiggler can smear out the density modulation but is ineffective in suppressing the growth of energy modulation accumulated in the early part of the machine. In Sec. III, we derive the gain suppression formula using a laser heater with an arbitrary transverse laser spot size. The electron energy profile generated from a laser heater with a laser spot size large compared to the transverse size of the electron beam deviates significantly from a Gaussian distribution and is not effective in smearing the short-wavelength microbunching. With a laser spot size matched to the transverse size of the electron beam, the laser heater generates a nearly Gaussian energy distribution and can be used to minimize the instability effects. The conceptual design of a laser heater embedded in a magnetic chicane is described in Sec. IV. Summaries and concluding remarks are given in Sec. V. 


\section{MICROBUNCHING INSTABILITY INCLUDING LSC}

The mechanism for microbunching instability is similar to that in a klystron amplifier [4]. A high-brightness electron beam with a small amount of longitudinal density modulation can create self-fields that lead to beam energy modulation. Since a magnetic bunch compressor (usually a chicane) introduces path length dependence on energy, the induced energy modulation is then converted to additional density modulation that can be much larger than the initial density modulation. This amplification process (the gain in microbunching) is accompanied by a growth of energy modulation and a possible growth of emittance if significant energy modulation is induced in a dispersive region such as the chicane. Thus, the instability can be harmful to FEL performance, which depends critically on the high quality of the electron beam.

The self-fields generated by the beam in the accelerator are characterized by a longitudinal impedance $Z(k)$ in the frequency domain, where $k=2 \pi / \lambda$ and $\lambda$ is the modulation wavelength. The microbunching instability driven by CSR and linac wakefields has been studied for the LCLS accelerator [10, 11]. To include the longitudinal space charge field, we use a round beam model with an uniform transverse cross section. The free-space LSC impedance per unit length is $[12,13]$

$$
\begin{aligned}
Z_{\mathrm{LSC}}(k) & =\frac{i Z_{0}}{\pi k r_{b}^{2}}\left[1-\frac{k r_{b}}{\gamma} K_{1}\left(\frac{k r_{b}}{\gamma}\right)\right] \\
& \approx \begin{cases}\frac{i Z_{0}}{\pi k r_{b}^{2}}, & \frac{k r_{b}}{\gamma} \gg 1, \\
\frac{i Z_{0} k}{4 \pi \gamma^{2}}\left(1+2 \ln \frac{\gamma}{r_{b} k}\right), & \frac{k r_{b}}{\gamma} \ll 1,\end{cases}
\end{aligned}
$$

where $Z_{0}=377 \Omega$ is the free space impedance, $r_{b}$ is the radius of the beam cross section and is approximately the sum of rms beam sizes in both transverse planes, $\gamma$ is the electron energy in units of its rest mass $m c^{2}$, and $K_{1}$ is the modified Bessel function. Effects of the vacuum chamber are ignored for these very short modulation wavelengths. We have also neglected a small transverse variation of the LSC field that can contribute to a slight increase of the local energy spread. The two approximate expressions in Eq. (1) are valid in the low and high energy limits, respectively. The implementation of the LSC impedance in the numerical tracking code elegant [14] is described in Appendix A.

The initial electron density modulation is most likely caused by a high-frequency mod-

ulation on the drive laser that produces the electron beam from the photocathode. The 


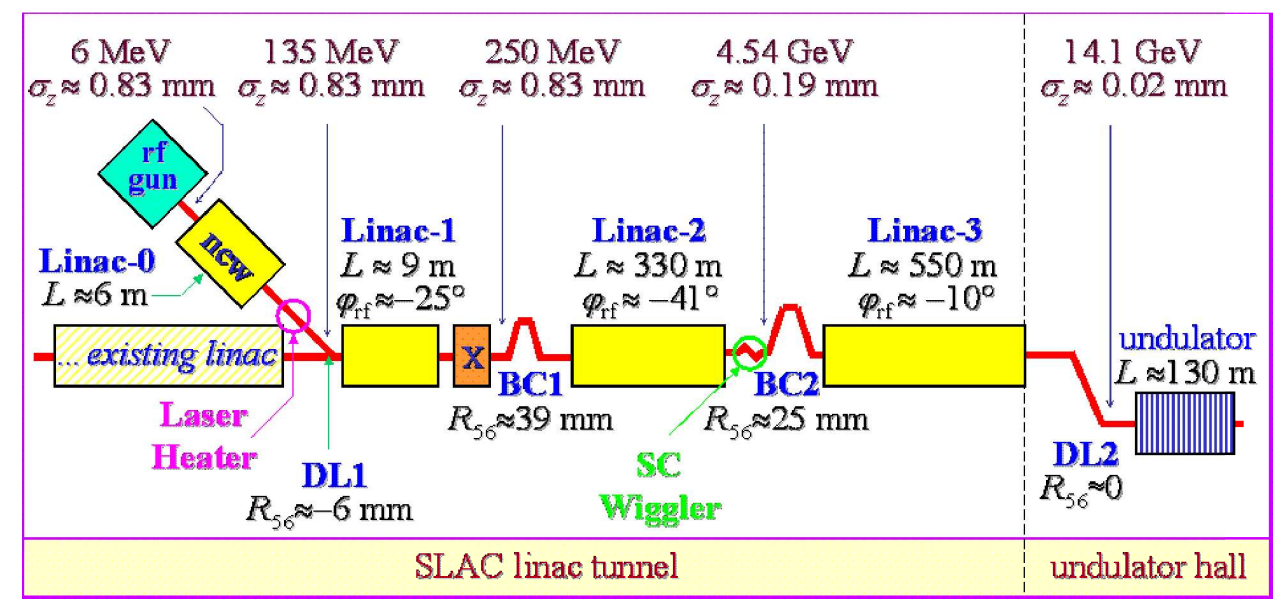

FIG. 1: (Color) Layout of the LCLS accelerator system with two Landau damping options: a superconducting (SC) wiggler at $4.5 \mathrm{GeV}$ or a laser heater at $135 \mathrm{MeV}$.

electrons repel each other in the higher density regions and initiate the space charge oscillation between density and energy modulations. The space charge oscillation frequency for a relativistic beam in a drift space is given by $[12,13]$

$$
\omega_{S C}=c\left[\frac{I_{0}}{\gamma^{3} I_{A}} k_{0} \frac{4 \pi\left|Z_{\mathrm{LSC}}\left(k_{0}\right)\right|}{Z_{0}}\right]^{1 / 2} \leq \frac{2 c}{r_{b}}\left(\frac{I_{0}}{\gamma^{3} I_{A}}\right)^{1 / 2} \equiv \omega_{p},
$$

where $I_{0}$ is the initial peak electron current, $I_{A} \approx 17 \mathrm{kA}$ is the Alfven current, $\lambda_{0}=2 \pi / k_{0}$ is the initial modulation wavelength, and $\omega_{p}$ is the plasma frequency when the transverse beam size is much larger than the reduced modulation wavelength in the beam's rest frame (i.e., $k_{0} r_{\mathrm{b}} / \gamma \gg 1$ ). Due to the space charge oscillation, the initial density modulation may be reduced at the expense of the increased energy modulation. Detailed modelling of spacecharge induced modulations in the LCLS photoinjector will be reported in Ref. [15]. Here, we consider the amplification of small beam density modulations starting from the end of the LCLS injector in order to focus on the microbunching instability in the main linac and to find its cure.

At the end of the LCLS photoinjector (at the energy $\gamma_{0} m c^{2}=135 \mathrm{MeV}$ ), the electrons are too relativistic to have any relative longitudinal motion in the linac. For example, for an initial beam current $I_{0}=120 \mathrm{~A}$, a transverse beam radius $r_{\mathrm{b}}=200 \mu \mathrm{m}$ and an initial modulation wavelength $\lambda_{0}=15 \mu \mathrm{m}$, the space charge oscillation period is $2 \pi c / \omega_{S C} \approx 100 \mathrm{~m}$ at $135 \mathrm{MeV}$, while the first linac section (Linac-1 in Fig. 1) accelerates the beam to $\gamma_{1} m c^{2}=$ $250 \mathrm{MeV}$ in $L=9 \mathrm{~m}$. Thus, the electron density modulation is frozen while the energy 
modulation is accumulated in the linac [7], i.e.,

$$
\Delta \gamma_{m}\left(k_{0}\right) \approx-\frac{I_{0} b_{0}\left(k_{0}\right)}{I_{A}} \int_{0}^{L} d s \frac{4 \pi Z\left(k_{0} ; s\right)}{Z_{0}},
$$

where the initial current spectrum is characterized by a bunching factor

$$
b_{0}\left(k_{0}\right)=\frac{1}{N e c} \int \Delta I_{0}\left(z_{0}\right) e^{-i k_{0} z_{0}} d z_{0},
$$

$N$ is the total number of electrons and $\Delta I_{0}\left(z_{0}\right)$ is the initial current variation as a function of the longitudinal coordinate $z_{0}$. (The head of the bunch is at $z_{0}>0$.)

To evaluate the gain of the density modulation $\left(\equiv\left|b_{f_{1}} / b_{0}\right|\right)$ after BC1 (the first bunchcompressor chicane shown in Fig. 1), we include both LSC and linac wakefield-induced energy modulation in Linac-1 as well as CSR in BC1 and DL1 (the first dog-leg transport line in Fig. 1). Assuming Gaussian distributions in energy and in transverse variables, we calculate the BC1 gain as shown in Fig. 2 using the formulas from Ref. [6]. The gain at very short wavelengths is dominated by the LSC impedance, which is inversely proportional to $\lambda_{0}$ at high beam energies. An important beam parameter for the instability is the initial uncorrelated energy spread. Both photoinjector simulations and measurements [16] show an rms value of about $3 \mathrm{keV}$ at about $1 \mathrm{nC}$ charge (i.e., $\sigma_{\gamma_{0}} \approx 0.006$ ). Thus, the smearing of microbunching from the uncorrelated energy spread across the chicane is not effective until

$$
\lambda_{0} \leq\left|\frac{2 \pi\left(R_{56}\right)_{1}}{1+h_{1}\left(R_{56}\right)_{1}}\right| \sigma_{\delta_{1}} \equiv \lambda_{c},
$$

where $h_{1} \approx-19.8 \mathrm{~m}^{-1}$ is the energy chirp, $\left(R_{56}\right)_{1} \approx 39 \mathrm{~mm}$ is the momentum compaction of $\mathrm{BC} 1$, and $\sigma_{\delta_{1}}=\sigma_{\gamma_{0}} / \gamma_{1} \approx 1.2 \times 10^{-5}$ is the relative energy spread just before BC1. As shown in Fig. 2, the gain peaks near $\lambda_{c} \approx 13 \mu \mathrm{m}$ and is exponentially suppressed at shorter wavelengths.

The large gain in density modulation after BC1 is capable of generating more energy modulation $\Delta \gamma_{m_{2}}$ in Linac-2 through LSC and linac wakefields, which can be estimated using Eq. (3) and is shown in Fig. 3 just prior to the entrance of BC2 (the second bunch-compressor chicane at the energy $\left.\gamma_{2} m c^{2}=4.54 \mathrm{GeV}\right)$. The intrinsic energy spread at this point is increased to about $\sigma_{\gamma_{1}} m c^{2}=\sigma_{\gamma_{0}} m c^{2} /\left|1+h_{1}\left(R_{56}\right)_{1}\right| \approx 13 \mathrm{keV}$ due to BC1 compression. Assuming the initial density modulation is very small so that $\left|b_{f_{1}}\right| \ll 1$ and

$$
\left|\frac{k_{0}\left(R_{56}\right)_{2} \Delta \gamma_{m_{2}}}{\left(1+h_{1}\left(R_{56}\right)_{1}\right)\left(1+h_{2}\left(R_{56}\right)_{2}\right) \gamma_{2}}\right| \ll 1,
$$




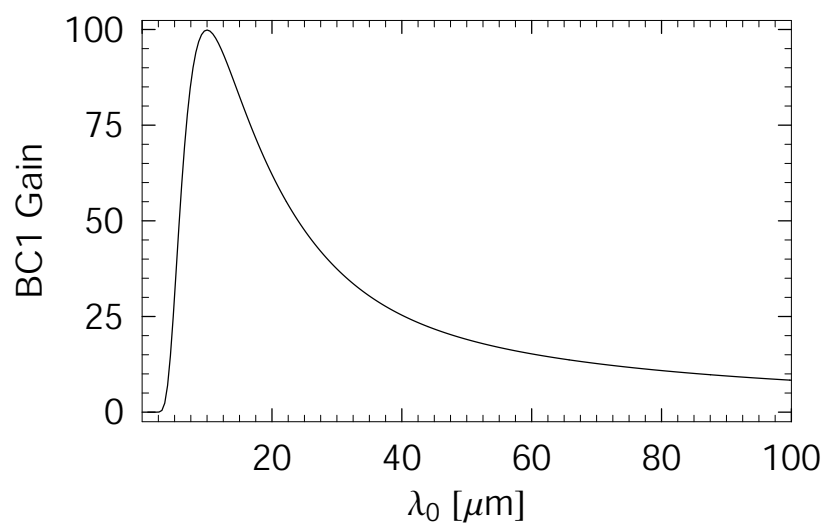

FIG. 2: Microbunching gain after BC1 as a function of the initial modulation wavelength $\lambda_{0}$.

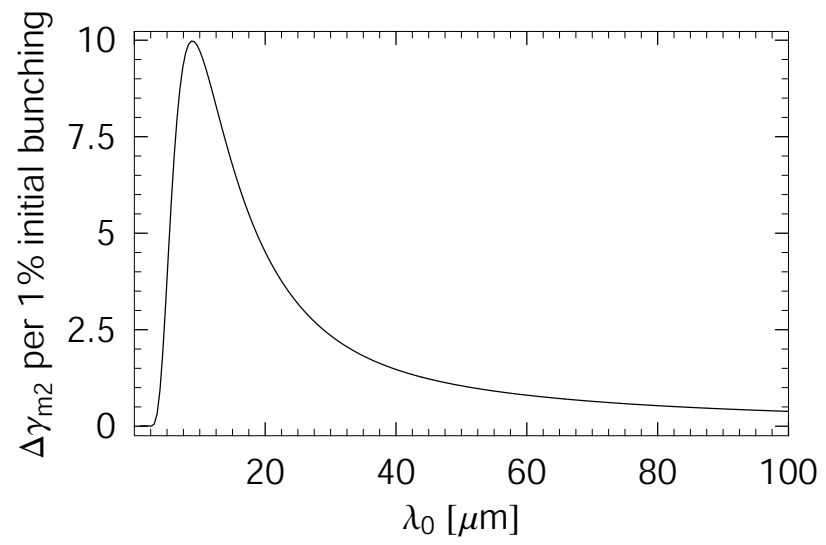

FIG. 3: Accumulated energy modulation $\Delta \gamma_{m_{2}}$ prior to BC2 as a function of the initial modulation wavelength $\lambda_{0}$.

we apply the linear theory again for BC2 to obtain the total gain in density modulation as shown in Fig. 4 (SC wiggler off), where we use the energy chirp $h_{2} \approx-34.3 \mathrm{~m}^{-1}$ and $\left(R_{56}\right)_{2} \approx$ $25 \mathrm{~mm}$ for this chicane. The extremely large gain of the system $(\sim 10000)$ indicates that even the electron shot-noise fluctuations can be strongly amplified. Note that the intrinsic energy spread after BC2 compression (with a compression factor $\left|1+h_{2}\left(R_{56}\right)_{2}\right|^{-1} \approx 7$ ) is increased to about $13 \mathrm{keV} \times 7 \approx 90 \mathrm{keV}$, equivalent to a relative rms energy spread $\sigma_{\delta_{f}}<1 \times 10^{-5}$ at the final LCLS energy $\gamma_{3} m c^{2} \approx 14 \mathrm{GeV}$.

The very large gain in density modulation at these short wavelengths can be suppressed by increasing the uncorrelated energy spread of the electron beam. Since the FEL parameter is about $5 \times 10^{-4}$ for the LCLS when the fundamental radiation wavelength is $1.5 \AA$ (see Table I), a factor of 10 to 15 increase in uncorrelated energy spread has a rather minimal 


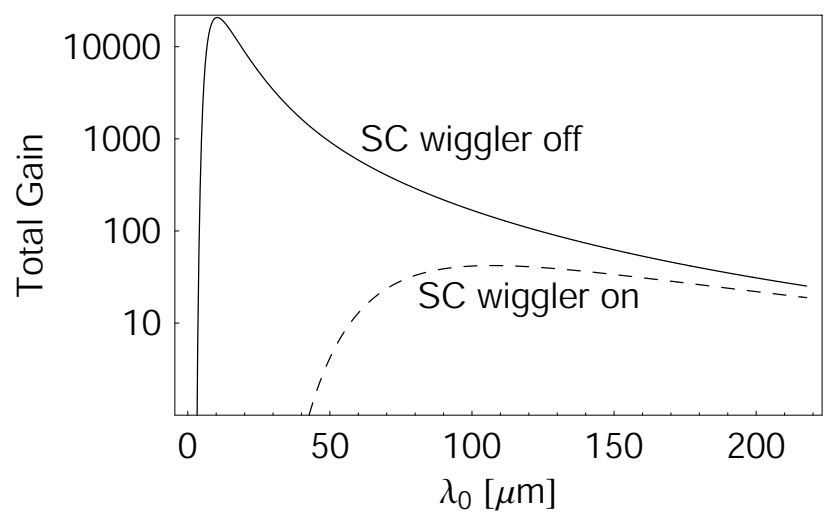

FIG. 4: Microbunching gain after BC2 as a function of the initial modulation wavelength $\lambda_{0}$ with the SC wiggler off (solid) and on (dashed).

TABLE I: Main parameters for the LCLS FEL.

\begin{tabular}{|lcc|}
\hline Parameter & Symbol & Value \\
\hline electron energy & $\gamma_{3} m c^{2}$ & $14.1 \mathrm{GeV}$ \\
bunch charge & $Q$ & $1 \mathrm{nC}$ \\
bunch current & $I_{f_{2}}$ & $3.4 \mathrm{kA}$ \\
transverse norm. emittance $\varepsilon_{x, y}^{n}$ & $1 \mu \mathrm{m}$ \\
average beta function & $\beta_{x, y}$ & $25 \mathrm{~m}$ \\
undulator period & $\lambda_{u}$ & $0.03 \mathrm{~m}$ \\
undulator field & $B$ & $1.3 \mathrm{~T}$ \\
undulator parameter & $K$ & 3.64 \\
undulator length & $L_{u}$ & $100 \mathrm{~m}$ \\
FEL wavelength & $\lambda_{r}$ & $1.5 \AA$ \\
FEL parameter & $\rho$ & $4.8 \times 10^{-4}$ \\
\hline
\end{tabular}

impact on the FEL performance. Using Table I and Xie's fitting formula [17], we plot the FEL power gain length as a function of the uncorrelated energy spread $\sigma_{\delta_{f}}$ at the undulator entrance in Fig. 5. At $\sigma_{\delta_{f}}=1 \times 10^{-4}$, the power gain length is increased by only $4 \%$. Taking into account that quantum fluctuations of spontaneous radiation in a 100-m undulator can increase the rms energy spread to $\sim 2 \times 10^{-4}$ [1], the average power gain length is almost independent of the initial energy spread up to $1 \times 10^{-4}$. However, for $\sigma_{\delta_{f}}>1 \times 10^{-4}$, the 


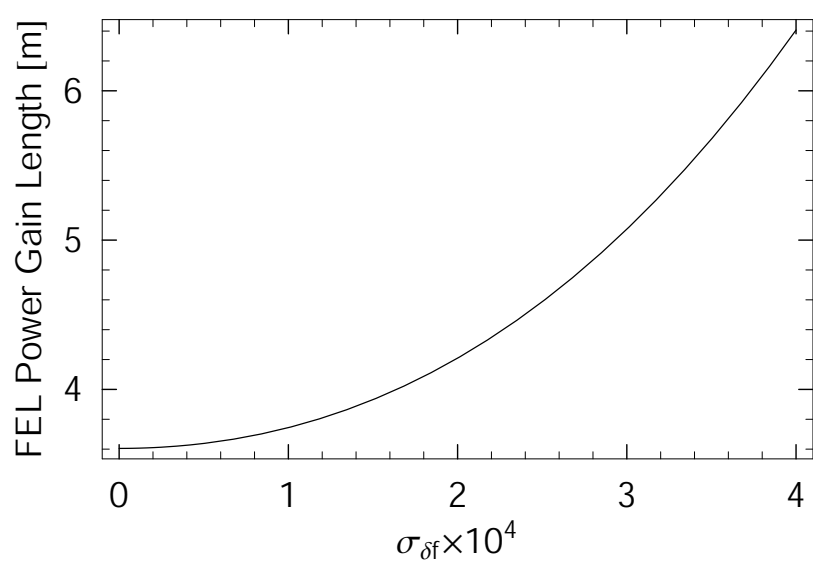

FIG. 5: LCLS FEL power gain length as a function of the uncorrelated energy spread $\sigma_{\delta_{f}}$ at the undulator entrance at $14 \mathrm{GeV}$.

FEL gain length and hence the saturation length starts to increase much faster. Thus, the tolerable rms energy spread at the undulator entrance is about $1 \times 10^{-4}$ or $1.4 \mathrm{MeV}$.

One way to increase the uncorrelated energy spread is to use a SC wiggler prior to $\mathrm{BC} 2$ [1]. As a result of incoherent synchrotron radiation induced in the wiggler, the rms energy spread before BC2 increases from $13 \mathrm{keV}$ to $\sigma_{\gamma_{2}} m c^{2}=170 \mathrm{keV}$, and the total gain in density modulations after BC2 is strongly suppressed for $\lambda_{0}<100 \mu \mathrm{m}$ as shown in Fig. 4. However, the wiggler does not affect the high-frequency energy modulation already accumulated before $\mathrm{BC} 2$, which can be temporally smeared in $\mathrm{BC} 2$ and increase the local energy spread on the scale of the FEL slippage length (slice energy spread). Starting with $\pm 1 \%$ initial density modulation at various wavelengths and tracking a few million particles in elegant from the injector end to the undulator entrance in presence of the $\mathrm{SC}$ wiggler, the slice energy spread in the bunch core (excluding head and tail) is much higher than the FEL limit $\left(\equiv 1 \times 10^{-4}\right)$ as shown in Fig. 6. If we assume that $\left(R_{56}\right)_{2}$ in BC2 completely washes out the induced short-wavelength energy modulations in Linac-2, the effective rms energy spread at the undulator entrance can be estimated as

$$
\sigma_{\delta_{f}} \approx \frac{1}{\gamma_{3}\left|1+h_{2}\left(R_{56}\right)_{2}\right|} \sqrt{\frac{\left(\Delta \gamma_{m_{2}}\right)^{2}}{2}+\sigma_{\gamma_{2}}^{2}} .
$$

Figure 6 shows that Eq. (7) agrees well with the simulation results. In this case, the uncorrelated energy spread increased by the $\mathrm{SC}$ wiggler is too late in the beam line to prevent the growth of LSC-induced density and energy modulations in the early-stage of acceleration and compression (in Linac-1,2 and BC1). 


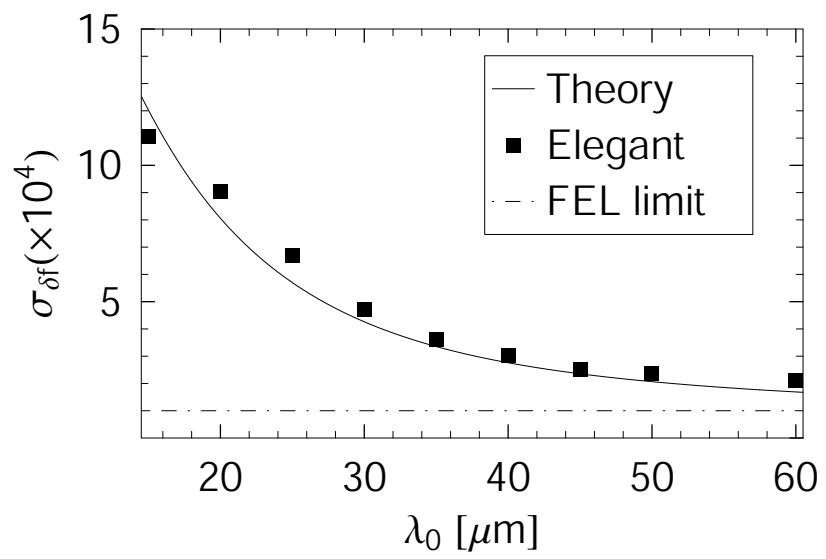

FIG. 6: Slice rms energy spread $\sigma_{\delta_{f}}$ at the undulator entrance at $14 \mathrm{GeV}$ for $1 \%$ initial density modulation at different wavelengths (SC wiggler on, no laser heater).

\section{GAIN SUPPRESSION WITH A LASER HEATER}

The above considerations indicate that the initial short-wavelength density modulation should be less than $10^{-3}$ even with the SC wiggler, or the gain of density modulation in $\mathrm{BC} 1$ should be strongly suppressed by increasing the local energy spread before any compression. At energies less than about $1 \mathrm{GeV}$, uncorrelated energy spread cannot be easily increased by quantum fluctuations of synchrotron radiation. Nevertheless, resonant laser-electron interaction in a short undulator induces rapid energy modulation at the optical frequency, which can be used as an effective energy spread for beam "heating" [7, 9]. In this section, we study the gain suppression using such a laser heater and discuss the role of the transverse laser profile in smearing out the microbunching.

Suppose a fundamental Gaussian mode laser co-propagates with a round electron beam at the energy $\gamma_{0} m c^{2}$ in an undulator of length $L_{u}$, which is short compared to both the Rayleigh length $Z_{R}$ of the laser and the beta functions $\beta_{x, y}$ of the electrons. The laser wavelength $\lambda_{L}$ satisfies the resonant condition given by $\lambda_{L}=\lambda_{u}\left(1+K^{2} / 2\right) /\left(2 \gamma_{0}^{2}\right)$, where $\lambda_{u}$ is the undulator period and $K$ is the undulator strength parameter. Neglecting small changes in laser and electron beam sizes during the resonant interaction, we obtain the amplitude of the FEL energy modulation as (see, e.g. [18])

$$
\Delta \gamma_{L}(r)=\sqrt{\frac{P_{L}}{P_{0}}} \frac{K L_{u}}{\gamma_{0} \sigma_{r}}\left[J_{0}\left(\frac{K^{2}}{4+2 K^{2}}\right)-J_{1}\left(\frac{K^{2}}{4+2 K^{2}}\right)\right] \exp \left(-\frac{r^{2}}{4 \sigma_{r}^{2}}\right),
$$

where $P_{L}$ is the peak laser power, $P_{0}=I_{A} m c^{2} / e \approx 8.7 \mathrm{GW}, J_{0,1}$ are the Bessel functions, $r$ is 
TABLE II: Main parameters for the LCLS laser heater.

\begin{tabular}{|llc|}
\hline Parameter & Symbol & Value \\
\hline electron energy & $\gamma_{0} m c^{2}$ & $135 \mathrm{MeV}$ \\
average beta function & $\beta_{x, y}$ & $10 \mathrm{~m}$ \\
transverse rms beam size & $\sigma_{x, y}$ & $190 \mu \mathrm{m}$ \\
undulator period & $\lambda_{u}$ & $0.05 \mathrm{~m}$ \\
undulator field & $B$ & $0.33 \mathrm{~T}$ \\
undulator parameter & $K$ & 1.56 \\
undulator length & $L_{u}$ & $0.5 \mathrm{~m}$ \\
laser wavelength & $\lambda_{L}$ & $800 \mathrm{~nm}$ \\
laser rms spot size & $\sigma_{r}$ & $1.5 \mathrm{~mm}(175 \mu \mathrm{m})$ \\
laser peak power & $P_{L}$ & $37 \mathrm{MW}(1.2 \mathrm{MW})$ \\
Rayleigh range & $Z_{R}$ & $44 \mathrm{~m}(0.6 \mathrm{~m})$ \\
maximum energy modulation & $\Delta \gamma_{L}(0) m c^{2}$ & $55 \mathrm{keV}(80 \mathrm{keV})$ \\
rms local energy spread & $\sigma_{\gamma_{L}} m c^{2}$ & $40 \mathrm{keV}$ \\
\hline
\end{tabular}

the radial position of the electron, and $\sigma_{r}$ is the rms laser spot size in the undulator. Table II lists the main laser heater parameters under design at the end of the LCLS photoinjector (see Fig. 1). Two sets of laser spot size and peak power are considered, both of which increase the rms energy spread from $3 \mathrm{keV}$ to about $40 \mathrm{keV}$. After a total compression factor of about 30 , the slice rms energy spread should be about $1.2 \mathrm{MeV}$ or $\sigma_{\delta_{f}} \approx 0.9 \times 10^{-4}$ at the undulator entrance (at $14 \mathrm{GeV}$ ) in the absence of impedance effects. The necessary laser power for the large laser spot size $\sigma_{r}=1.5 \mathrm{~mm} \gg \sigma_{x}$ is still a small fraction of the available power of the Ti-Sapphire laser that drives the photocathode rf gun and hence can be extracted from it.

The electron distribution is modified after the laser-electron interaction. Assuming initially Gaussian distributions in energy and in transverse coordinates, the electron distribution function, including the transverse dependence, becomes

$$
f_{0}\left(z_{0}, \Delta \gamma_{0}, r\right)=\frac{I_{0}}{e c \sqrt{2 \pi} \sigma_{\gamma_{0}}} \exp \left[-\frac{\left(\Delta \gamma_{0}-\Delta \gamma_{L}(r) \sin k_{L} z_{0}\right)^{2}}{2 \sigma_{\gamma_{0}}^{2}}\right] \times \frac{1}{2 \pi \sigma_{x}^{2}} \exp \left(-\frac{r^{2}}{2 \sigma_{x}^{2}}\right)
$$

where $k_{L}=2 \pi / \lambda_{L}$ and $\sigma_{x}\left(=\sigma_{y}\right)$ is the rms electron beam size in the transverse plane. Integrating this distribution function over transverse and longitudinal coordinates, we obtain 


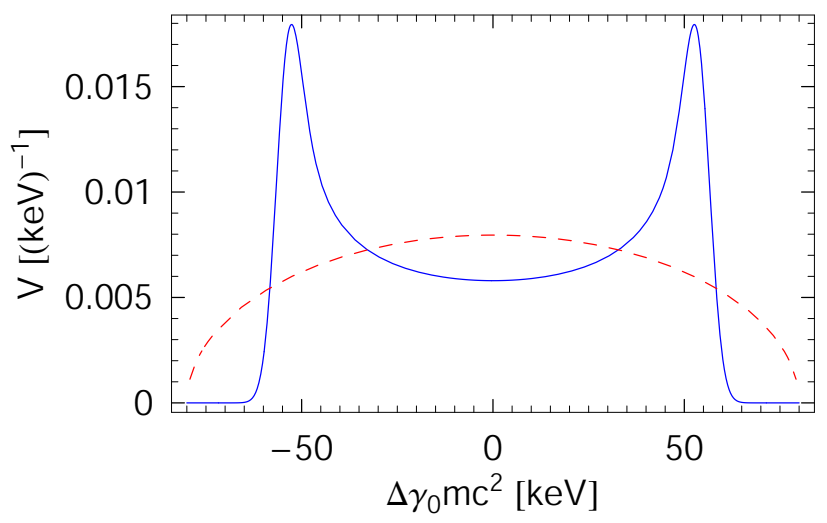

FIG. 7: (Color) Electron energy distribution after the laser heater for a large laser spot (blue solid) and for a matched laser spot (red dashed). The laser powers are given in Table II so that the rms energy spread $\sigma_{\gamma_{L}} m c^{2} \approx 40 \mathrm{keV}$ for both distributions.

the modified energy distribution

$$
\begin{aligned}
V\left(\Delta \gamma_{0}\right) & =2 \pi \int r d r \int d z_{0} f_{0}\left(z_{0}, \Delta \gamma_{0}, r\right) \\
& =\frac{1}{\pi \sigma_{x}^{2} \sqrt{2 \pi} \sigma_{\gamma_{0}}} \int r d r \exp \left(-\frac{r^{2}}{2 \sigma_{x}^{2}}\right) \int \frac{d \xi}{\sqrt{\Delta \gamma_{L}(r)^{2}-\left(\Delta \gamma_{0}-\xi\right)^{2}}} \exp \left(-\frac{\xi^{2}}{2 \sigma_{\gamma_{0}}^{2}}\right) .
\end{aligned}
$$

Using laser heater parameters from Table II, we plot the energy distribution in Fig. 7 for $\sigma_{r} \gg \sigma_{x}$ (when the laser spot size is much larger than the electron beam size) and $\sigma_{r} \approx \sigma_{x}$ (when the laser spot size is matched to the e-beam size). Although a large laser spot size is less sensitive to beam position jitter in the transverse planes, the energy modulation amplitude is almost the same for all electrons, and the energy profile is a double-horn distribution as shown in Fig. 7 . The two sharp spikes at $\Delta \gamma \approx \pm \Delta \gamma_{L}(0)$ act like two separate cold beams that do not contribute much to suppressing the instability. For a laser spot comparable to the e-beam size, the off-axis electrons experience smaller modulation with smaller laser field than the on-axis ones. As a result, the "heating" is more uniform in terms of the energy spread. As shown in Fig. 7, the energy profile predicted from Eq. (10) for $\sigma_{r} \approx \sigma_{x}$ is similar to a Gaussian distribution, and we expect more effective Landau damping.

Let us first consider the microbunching gain of a single bunch compressor due to its upstream impedances (LSC and linac wakefields) in presence of a laser heater. Appendix B gives the gain formula for an arbitrary energy distribution. Inserting Eq. (10) into Eq. (B5), 
we obtain

$$
G=\left|\frac{b_{f}}{b_{0}}\right| \approx \frac{I_{0}}{\gamma I_{A}}\left|k_{f} R_{56} \int_{0}^{L} d s \frac{4 \pi Z\left(k_{0} ; s\right)}{Z_{0}}\right| \exp \left(-\frac{1}{2} k_{f}^{2} R_{56}^{2} \sigma_{\delta}^{2}\right) S_{L}\left(k_{f} R_{56} \delta_{L}(0), \sigma_{r} / \sigma_{x}\right),
$$

where $k_{f}=k_{0} /\left|1+h R_{56}\right|$ is the compressed modulation wavenumber, and $\delta_{L}(0)=\Delta \gamma_{L}(0) / \gamma$ is the relative energy modulation amplitude at the energy $\gamma m c^{2}$ of the bunch compressor. Comparing with Eq. (B6), the gain suppression factor due to the laser heater is

$$
\begin{aligned}
S_{L}(A, B) & =\int R d R \exp \left(-\frac{R^{2}}{2}\right) J_{0}\left[A \exp \left(-\frac{R^{2}}{4 B^{2}}\right)\right]={ }_{1} F_{2}\left(B^{2} ; 1,1+B^{2} ;-\frac{A^{2}}{4}\right) \\
& = \begin{cases}J_{0}(A), & B \gg 1, \\
\frac{2 J_{1}(A)}{A}, & B=1 .\end{cases}
\end{aligned}
$$

Here ${ }_{1} F_{2}$ is the generalized hypergeometric function. For $|A| \gg 1$, the Bessel functions $J_{0,1}(A) \sim|A|^{-1 / 2}$. Thus, a laser heater with a large laser spot size $(B \gg 1)$ has $S_{L} \sim$ $\left|k_{f} R_{56} \delta_{L}(0)\right|^{-1 / 2}$ and suppresses the gain weakly, while a laser heater with a matched spot size $(B=1)$ has $S_{L} \sim\left|k_{f} R_{56} \delta_{L}(0)\right|^{-3 / 2}$ and is more effective at smearing the instability at short wavelengths.

The gain suppression factor, Eq. (12), can be approximately applied to the CSR microbunching in a chicane, which is also subject to emittance damping [4-6]. Thus, we can estimate the microbunching gain including LSC, CSR and linac wakefields in the LCLS using a laser heater. Figure 8 shows that the BC1 gain computed from the linear theory agrees reasonably with elegant simulations using two sets of laser spot size and peak power given in Table II. Note that a particle-tracking code for the resonant laser-electron interaction is used to simulate the heating process prior to the elegant runs. The oscillatory behavior of the gain spectrum is due to the nearly hard-edge cutoff in the energy profile of a laser-heated beam (see Fig. 7), but the gain from a large laser spot $\left(\sigma_{r}=1.5 \mathrm{~mm}\right)$ is clearly much larger than that from a matched laser spot $\left(\sigma_{r}=175 \mu \mathrm{m}\right)$ at short wavelengths.

The knowledge of the gain spectrum together with the initial bunching spectrum determine the compressed current profile $I_{f}(z)$. The variance of the current profile is

$$
\frac{\int d z\left|\Delta I_{f}(z)\right|^{2}}{\Delta z_{f} I_{f}^{2}}=\frac{\Delta z_{f}}{2 \pi} \int d k_{f}\left|b_{f}\left(k_{f}\right)\right|^{2}=\frac{\Delta z_{0}}{2 \pi} \int d k_{0}\left|G\left(k_{0}\right) b_{0}\left(k_{0}\right)\right|^{2},
$$

where $\Delta I_{f}(z)$ is the variation of the compressed current from its average value, $\Delta z_{f}$ and $\Delta z_{0}$ are the final and initial bunch lengths (FWHM), respectively, and we have used Parseval's 


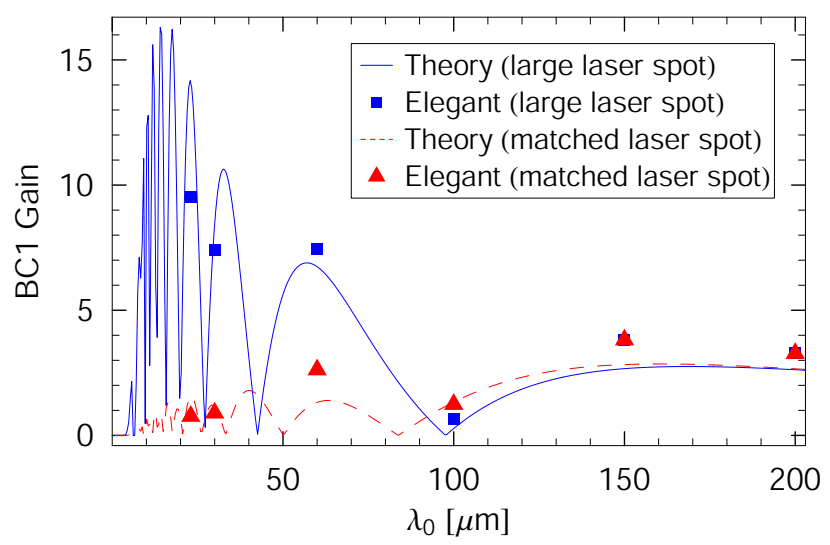

FIG. 8: (Color) Microbunching gain after BC1 as a function of the initial modulation wavelength $\lambda_{0}$ for a laser heater with a large laser spot (blue) and with a matched laser spot (red).

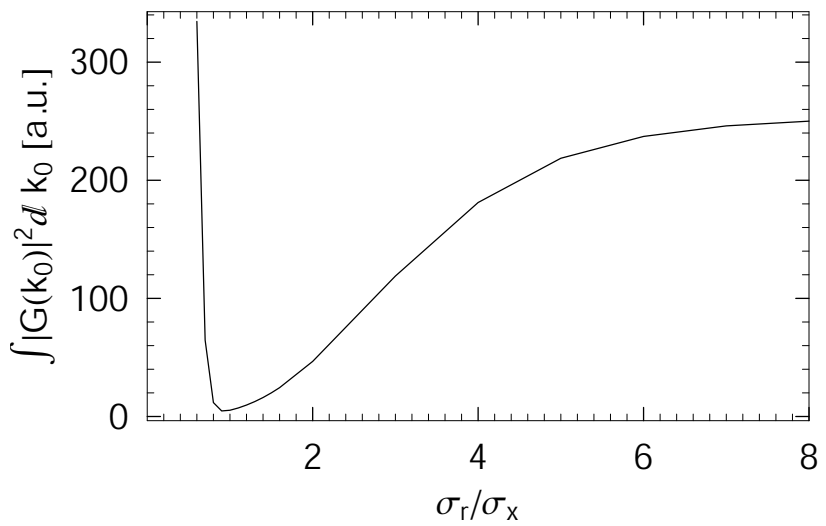

FIG. 9: Variance of the current profile after $\mathrm{BC} 1$ as a function of the spot size from the heater laser for a white noise bunching spectrum.

relation between the Fourier transformation pair $\left(z, k_{f}\right)$. Assuming an initially "white" bunching spectrum (i.e., $\left\langle\left|b\left(k_{0}\right)\right|^{2}\right\rangle=$ constant) and taking $\Delta \gamma_{L}(0) m c^{2}=80 \mathrm{keV}$, we integrate the right hand side of Eq. (13) for an arbitrary laser spot size. Fig. 9 shows that the current variance is minimized after $\mathrm{BC} 1$ for $\sigma_{r} \approx \sigma_{x}$, indicating the optimal heating for a laser with its spot size matched to the electron beam.

Figure 10 shows the total gain after BC2 in presence of a laser heater computed from the linear theory along with elegant simulation results. The theoretical gain at short wavelengths can still be very high $(\sim 300)$ for a laser heater with a large spot size because of its ineffective Landau damping at these wavelengths. However, comparison with simulations for $\lambda_{0} \leq$ $60 \mu \mathrm{m}$ is difficult due to both numerical noise and nonlinear behavior. For instance, the 


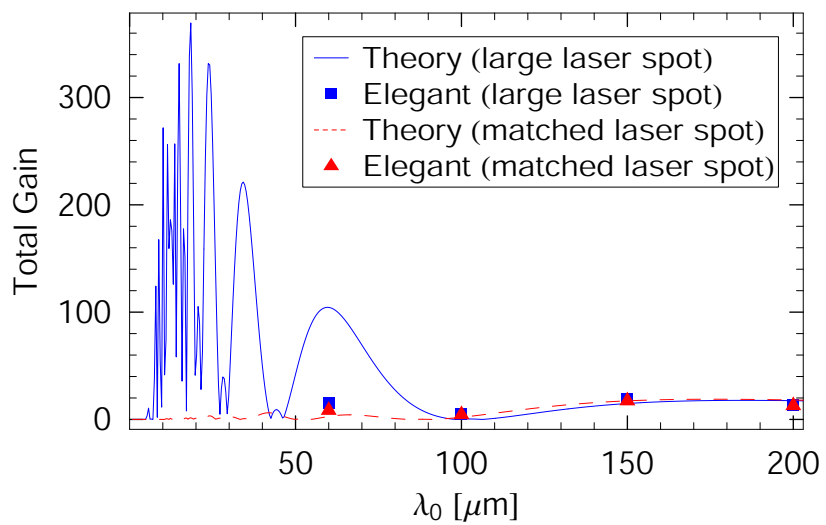

FIG. 10: (Color) Microbunching gain after BC2 as a function of the initial modulation wavelength $\lambda_{0}$ for a laser heater with a large laser spot (blue) and with a matched laser spot (red).

total gain with a large laser spot at $\lambda_{0}=60 \mu \mathrm{m}$ from elegant is much smaller than the linear theory (see Fig. 10) because the linear approximation breaks down for the initial 1\% density modulation used in the simulation (i.e., Eq. (6) is not satisfied). In this case, the simulated gain is reduced as the density modulation after BC2 is not sinusoidal, but the local energy spread can still increase as a result of the distorted longitudinal phase space (see Fig. 11). Figure 12 shows the slice energy spread of the bunch core at the undulator entrance without a laser heater (already discussed in Sec. II) and in presence of a laser heater with two different spot sizes. Thus, a laser heater with a large laser spot allows the growth of short-wavelength modulations that increases the slice energy spread at the undulator entrance, while a laser heater with a matched laser spot effectively suppresses the instability and does not change the slice energy spread above the design goal (about $\left.0.9 \times 10^{-4}\right)$.

\section{LASER HEATER DESIGN}

As shown in Fig. 13, the LCLS laser heater consists of a 50-cm long, 5-cm period undulator located at the center of a small horizontal magnetic chicane in order to allow convenient laser-electron interaction with no crossing angle. The electron and laser beam parameters are listed in Table II. In addition to easy optical access, the chicane provides a useful temporal washing effect that completely smears the laser-induced 800-nm energy modulation, resulting in a random energy spread with no temporal structure. This smearing occurs because the path length from chicane center (where the energy modulation is induced) to 


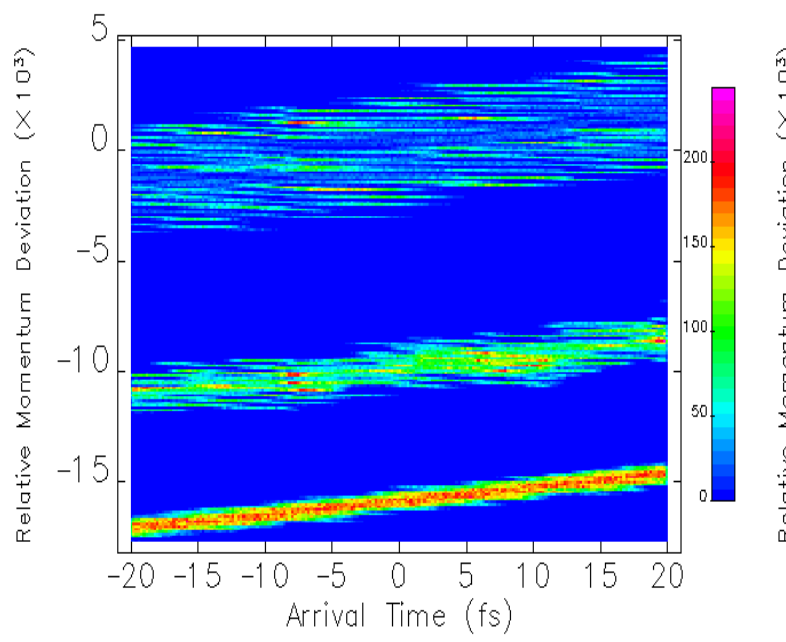

(a)End of $\mathrm{BC} 2$ at $4.5 \mathrm{GeV}$

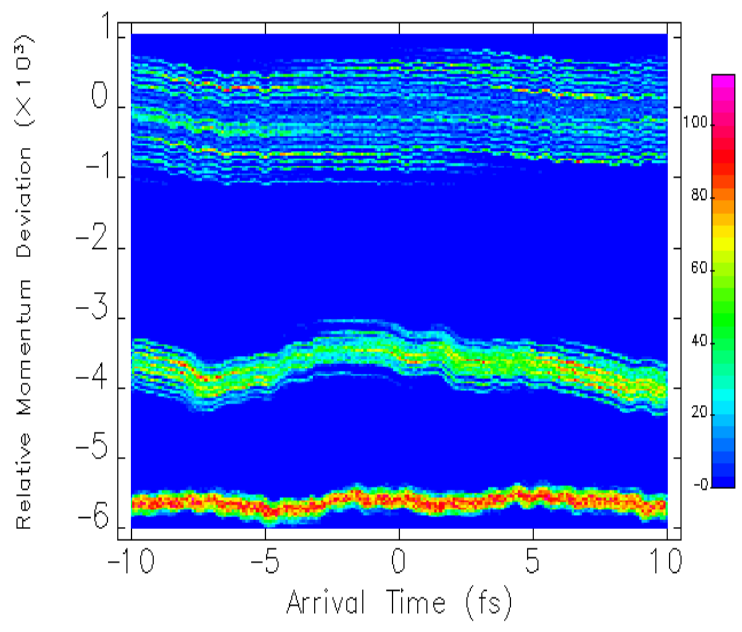

(b) Undulator entrance at $14 \mathrm{GeV}$

FIG. 11: (Color) Central portion of the longitudinal phase space without a laser heater (up), in presence of a laser heater with $\sigma_{r}=1.5 \mathrm{~mm}$ (middle) and with $\sigma_{r}=175 \mu \mathrm{m}$ (down). Curves offset vertically for clarity. Simulations are seeded with $1 \%$ initial density modulation at $\lambda_{0}=30 \mu \mathrm{m}$.

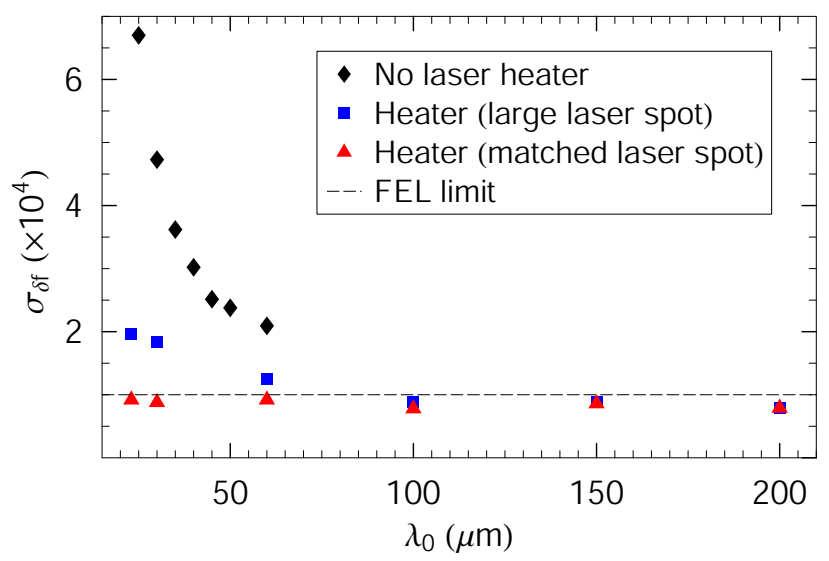

FIG. 12: (Color) Slice rms energy spread $\sigma_{\delta_{f}}$ at the undulator entrance at $14 \mathrm{GeV}$ for $1 \%$ initial density modulation without a laser heater (black), in presence of a laser heater with a large spot size (blue) and with a matched spot size (red).

chicane end depends on the electron's horizontal angle, $x^{\prime}$. For a symmetric chicane with momentum dispersion $\eta$ at its center, and no angular dispersion, the relevant path length coefficients across the half-chicane are $R_{52}=-\eta$ and $R_{51}=0$. Therefore, the rms temporal 
(or longitudinal) smearing is given by

$$
\Delta \sigma_{z}=\left|R_{52}\right| \sigma_{x^{\prime}}=|\eta| \sigma_{x^{\prime}} \gg \lambda_{L}
$$

where $\sigma_{x^{\prime}}(\approx 20 \mu \mathrm{rad})$ is the rms angular spread of the electron beam at the center of the chicane, and $\lambda_{L}\left(\equiv \lambda_{L} /(2 \pi) \approx 127 \mathrm{~nm}\right)$ is the reduced wavelength of the laser. With a dispersion value of $\eta \approx 20 \mathrm{~mm}$, the rms temporal smearing is $400 \mathrm{~nm}$, which is large compared to the reduced wavelength, $\lambda_{L}$. Thus, the 800-nm energy modulation structure is completed removed before the $R_{56}$ of this chicane $(\approx 3 \mathrm{~mm})$ or DL1 $(\approx-6 \mathrm{~mm})$ turn it into any density modulation.

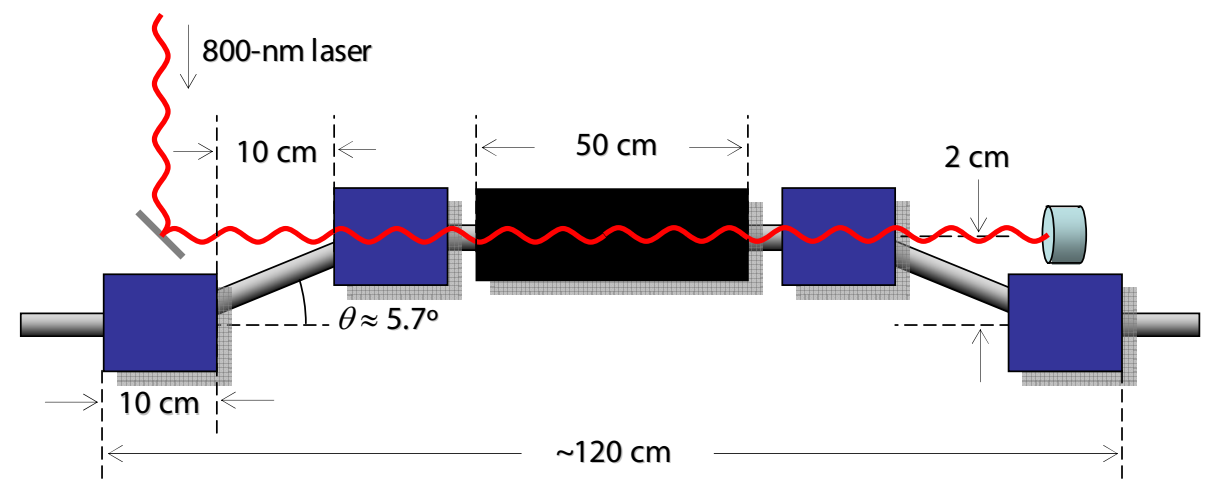

FIG. 13: (Color) Layout of the LCLS laser heater inside a magnetic chicane at $135 \mathrm{MeV}$.

Finally, the induced energy spread at the center of the chicane causes some horizontal emittance growth. This can be estimated by comparing the heater-induced energy spread, $\sigma_{\gamma_{L}} / \gamma_{0}$, multiplied by the dispersion, $\eta$, to the nominal beam size, $\sigma_{x}$, or

$$
\frac{\Delta \epsilon_{x}}{\epsilon_{x}} \approx \frac{1}{2}\left(\frac{\sigma_{\gamma_{L}} \eta}{\gamma_{0} \sigma_{x}}\right)^{2} .
$$

With $\sigma_{\gamma_{L}} / \gamma_{0}=0.04 / 135 \approx 3 \times 10^{-4}$ and $\sigma_{x} \approx 190 \mu \mathrm{m}$, the relative emittance growth is negligible $\left(\Delta \epsilon_{x} / \epsilon_{x}<0.1 \%\right)$.

\section{CONCLUSION}

Extremely bright electron beams are required to drive the FEL instability in the $\mathrm{x}$ ray wavelengths. However, accelerating and compressing a high-brightness electron beam inevitably introduces the microbunching instability driven by collective effects in the accelerator. Since an x-ray FEL such as the LCLS is not sensitive to the very small local energy 
spread of the beam generated from the photocathode rf gun, increasing it within the FEL tolerance damps the instability significantly without affecting the FEL gain length. In this paper, both analytical and numerical approaches are used to investigate different Landau damping options in the LCLS. We find that a laser heater with the laser transverse spot size equal approximately to the transverse size of the electron beam is most effective in suppressing the growth of both density and energy modulations in a wide spectral range. Such a laser heater also allows the flexible control of the slice energy spread to explore the FEL physics. Thus, the results of this study will be important for designs of future x-ray FEL projects that are based on similar electron beam and accelerator technologies.

\section{Acknowledgments}

We would like to thank K. Bane, P. Bolton, R. Carr, D. Dowell, W. Fawley, J. Galayda, K.-J. Kim, Y.-J. Kim, S. Krinsky, E. Schneidmiller, T. Shaftan, and T. Smith for useful discussions. This work was supported by Department of Energy contracts DE-AC0376SF00515 and W-31-109-ENG-38.

\section{APPENDIX A: LONGITUDINAL SPACE CHARGE SIMULATION WITH $E L E$ -} GANT

Elegant supplies two beamline elements that simulate longitudinal space charge. One is a drift element and the other an rf cavity element that also includes structure wakefields. The exact form of the longitudinal space charge in Eq. (1) is used in a kick-drift-kick (or kickaccelerate-kick) algorithm. The distance between kicks must be set properly to get a valid

result. This is satisfied if the distance is $L \ll c / \omega_{p}$, where $\omega_{p}$ is the plasma frequency given in Eq. (2). (For $I_{0}$, we use the maximum instantaneous current determined from a histogram of the particle arrival times at the kick location. This histogram is also used later in the algorithm. The number of bins is determined by the user.) The drift element automatically selects the drift distance, using $L=0.1 c / \omega_{p}$. The acceleration element requires the user to specify the number of parts to split the cavity into, and simply checks that $L \leq 0.1 c / \omega_{p}$. For the case with acceleration, we must impose an additional condition. In particular, we require $L \leq 0.1 \gamma /(d \gamma / d s)$. This ensures that the momentum does not change too much between 
kicks. For a Gaussian or a parabolic transverse beam distribution, we fit $r_{b} \approx 1.7\left(\sigma_{x}+\sigma_{y}\right) / 2$ in Eq. (1), where $\sigma_{x}$ and $\sigma_{y}$ are the rms beam sizes in the transverse planes.

Having computed the impedance, we next take the fast Fourier transformation (FFT) of the current histogram. This is optionally low-pass filtered to control noise. The cutoff frequency and slope of the filter are determined by the user. Generally, we choose the number of bins such that the frequencies of interest are less than $0.2 F_{n}$, where $F_{n}$ is the Nyquist frequency. We then use the low-pass filter to remove frequencies above $0.4 F_{n}$. Examination of FFTs of the current histograms provides guidance in this process. We have found this far more effective in controlling noise than using smoothing algorithms such as SavitzkyGolay, which in Fourier analysis are seen to do little more than put notch filters in at high frequencies.

The (filtered or unfiltered) FFT of the current is then multiplied by the impedance, and the result is inversely Fourier transformed. This gives the voltage as a function of bin in the original current histogram. We apply this voltage to each particle, with interpolation between bins to make a smoother result.

\section{APPENDIX B: MICROBUNCHING GAIN FOR AN ARBITRARY ENERGY DISTRIBUTION}

We generalize the microbunching gain of a bunch compressor due to its upstream impedance [4] to an arbitrary energy distribution. A beam with an initial density modulation (quantified by $b_{0}\left(k_{0}\right)$ in Eq. (4)) induces an energy modulation $\Delta \gamma_{m}$ (given in Eq. (3)) in the beam line before arriving at the bunch compressor. Thus, its longitudinal distribution function becomes

$$
F\left(z_{0}, \delta\right)=F_{0}\left(z_{0}, \delta-h z_{0}-\delta_{m}\left(z_{0}\right)\right),
$$

where $\delta=\Delta \gamma / \gamma$ is the normalized energy variable, $\gamma m c^{2}$ is the beam energy at the bunch compressor, $F_{0}\left(z_{0}, \delta_{0}=\Delta \gamma_{0} / \gamma\right)$ is the initial longitudinal distribution, $h z_{0}$ is the linear energy chirp before compression, and $\delta_{m}=\Delta \gamma_{m} / \gamma$ is the relative energy modulation.

The bunch compressor introduces a path length dependence on energy through its momentum compaction $R_{56}$, i.e., the longitudinal position of the electron with a relative energy deviation $\delta$ becomes

$$
z=z_{0}+R_{56} \delta=z_{0}+R_{56}\left[\delta_{0}+h z_{0}+\delta_{m}\left(z_{0}\right)\right] .
$$


Thus, the energy modulation is converted into additional density modulation at a compressed wavenumber $k_{f}$ given by

$$
b_{f}\left(k_{f}\right)=\int d z d \delta e^{-i k_{f} z} F(z, \delta)=\int d z_{0} d \delta_{0} e^{-i k_{f} z_{0}-i k_{f} R_{56}\left[\delta_{0}+h z_{0}+\delta_{m}\left(z_{0}\right)\right]} F_{0}\left(z_{0}, \delta_{0}\right) .
$$

If the induced energy modulation is small such that $\left|k_{f} R_{56} \delta_{m}\right| \ll 1$, we expand Eq. (B3) to the linear order in $\delta_{m}$ and obtain

$$
b_{f}\left(k_{f}\right)=\left[b_{0}\left(k_{0}\right)-i k_{f} R_{56} \delta_{m}\left(k_{0}\right)\right] \int d \delta_{0} V\left(\delta_{0}\right) e^{-i k_{f} R_{56} \delta_{0}}
$$

where $k_{f}=k_{0} /\left(1+h R_{56}\right)$, and $V\left(\delta_{0}\right)$ is the initial beam energy distribution. Inserting Eq. (3) into Eq. (B4) and making the high-gain approximation (i.e., $\left|b_{f}\right| \gg\left|b_{0}\right|$ ), we obtain the gain for an arbitrary energy distribution

$$
G=\left|\frac{b_{f}}{b_{0}}\right| \approx \frac{I_{0}}{\gamma I_{A}}\left|k_{f} R_{56} \int_{0}^{L} d s \frac{4 \pi Z\left(k_{0} ; s\right)}{Z_{0}}\right| \int d \delta_{0} V\left(\delta_{0}\right) e^{-i k_{f} R_{56} \delta_{0}} .
$$

For a gaussian energy distribution with the rms relative energy spread $\sigma_{\delta}$, the gain in density modulation is [4]

$$
G \approx \frac{I_{0}}{\gamma I_{A}}\left|k_{f} R_{56} \int_{0}^{L} d s \frac{4 \pi Z\left(k_{0} ; s\right)}{Z_{0}}\right| \exp \left(-\frac{1}{2} k_{f}^{2} R_{56}^{2} \sigma_{\delta}^{2}\right) .
$$

[1] Linac Coherent Light Source (LCLS) Conceptual Design Report, SLAC-R-593, SLAC (2002).

[2] TESLA XFEL: First Stage of the X-ray Laser Laboratory (Technical Design Report, Supplement), TESLA FEL 2002-09, DESY (2002).

[3] M. Borland, Y. Chae, P. Emma, J. Lewellen, V. Bharadwaj, W. Fawley, P. Krejcik, C. Limborg, S. Milton, H.-D. Nuhn, R. Soliday, and M. Woodley, Nuclear Instruments and Methods, Sec. A 483, 268 (2002).

[4] E. L. Saldin, E. A. Schneidmiller, and M. V. Yurkov, Nuclear Instruments and Methods, Sec. A 490, 1 (2002).

[5] S. Heifets, G. Stupakov, and S. Krinsky, Phys. Rev. ST Accel. Beams 5, 064401 (2002).

[6] Z. Huang and K.-J. Kim, Phys. Rev. ST Accel. Beams 5, 074401 (2002).

[7] E. L. Saldin, E. A. Schneidmiller, and M. V. Yurkov, Longitudinal space charge driven microbunching instability in TTF2 linac, TESLA-FEL-2003-02, DESY (2003). 
[8] T. Shaftan and Z. Huang, Experimental characterization of a space charge induced modulation in high-brightness electron beam, BNL-71491, BNL (2003).

[9] J. Galayda, private communication.

[10] Z. Huang, M. Borland, P. Emma, and K.-J. Kim, Nuclear Instruments and Methods, Sec. A 507, 318 (2003).

[11] Z. Huang, M. Borland, P. Emma, and K.-J. Kim, in Proceedings of the 2003 Particle Accelerator Conference (IEEE, Piscataway, NJ, 2003).

[12] J. Rosenzweig, C. Pellegrini, L. Serafini, C. Ternienden, and G. Travish, Space-charge oscillations in a self-modulated electron beam in multi-undulator free-electron lasers, TESLA-FEL96-15, DESY (1996).

[13] Z. Huang and T. Shaftan, in Proceedings of the 2003 Free-Electron Laser Conference, SLACPUB-9788, SLAC (2003).

[14] M. Borland, Elegant: A Flexible SDDS-Compliant Code for Accelerator Simulation, Advanced Photon Source LS-287, ANL (2000).

[15] C. Limborg et al., to be published.

[16] M. Hüning and H. Schlarb, in Proceedings of the 2003 Particle Accelerator Conference (IEEE, Piscataway, NJ, 2003).

[17] M. Xie, in Proceedings of the 1995 Particle Accelerator Conference (IEEE, Piscataway, NJ, 1995), p. 183.

[18] T. M. Tran and J. S. Wurtele, Comput. Phys. Commun. 54, 263 (1989). 